\title{
Rotating collapse of stellar iron cores in general relativity
}

\author{
C D Ott ${ }^{1,2}$, H Dimmelmeier ${ }^{3}$, A Marek ${ }^{3}$, H-T Janka ${ }^{3}$, B Zink ${ }^{4}$, I Hawke ${ }^{5}$ \\ and E Schnetter ${ }^{4}$ \\ ${ }^{1}$ Max-Planck-Institut für Gravitationsphysik, Albert-Einstein-Institut, Am Mühlenberg 1, \\ 14476 Potsdam, Germany \\ 2 JINA Postdoctoral Fellow, Department of Astronomy and Steward Observatory, The University \\ of Arizona, Tucson, AZ 85721, USA \\ ${ }^{3}$ Max-Planck-Institut für Astrophysik, Karl-Schwarzschild-Strasse 1, 85741 Garching, Germany \\ ${ }^{4}$ Center for Computation and Technology, Louisiana State University, 216 Johnston Hall, \\ Baton Rouge, LA 70803, USA \\ ${ }^{5}$ School of Mathematics, University of Southampton, Southampton SO17 1BJ, UK \\ E-mail: cott@as.arizona.edu
}

Received 20 December 2006, in final form 15 March 2007

Published 30 May 2007

Online at stacks.iop.org/CQG/24/S139

\begin{abstract}
We present results from the first $2+1$ and $3+1$ simulations of the collapse of rotating stellar iron cores in general relativity employing a finite-temperature equation of state and an approximate treatment of deleptonization during collapse. We compare full $3+1$ and conformally-flat spacetime evolution methods and find that the conformally-flat treatment is sufficiently accurate for the core-collapse supernova problem. We focus on the gravitational wave (GW) emission from rotating collapse, core bounce and early postbounce phases. Our results indicate that the GW signature of these phases is much more generic than previously estimated. In addition, we track the growth of a nonaxisymmetric instability of dominant $m=1$ character in two of our models that leads to prolonged narrow-band GW emission at $\sim 920-930 \mathrm{~Hz}$ over several tens of milliseconds.
\end{abstract}

PACS numbers: 04.25.Dm, 04.30.Db, 95.30.Sf, 97.60.Bw

(Some figures in this article are in colour only in the electronic version)

\section{Introduction}

For more than two decades astrophysicists have struggled to compute the gravitational wave (GW) signal produced by rotating stellar iron core collapse and the subsequent supernova evolution. Besides the coalescence of black hole and neutron star binaries, core-collapse 
events are considered to be among the most promising sources of detectable GWs. Theoretical predictions of the core-collapse supernova GW signature are still hampered by three major problems: (i) the rotational configuration prior to gravitational collapse is still uncertain since multi-D evolutionary calculations of rotating massive stars have not yet been performed; (ii) reliable waveform estimates require a general relativistic (GR) treatment, since both high densities and high velocities in combination with strong gravitational fields are encountered in this problem; and (iii) an adequate treatment of the nuclear equation of state (EOS) and the neutrino microphysics/radiative transfer is crucial for obtaining realistic collapse, bounce, and postbounce dynamics and waveforms. GW emission from core-collapse supernovae may arise from rotating collapse and bounce, postbounce neutrino-driven convection, anisotropic neutrino emission, nonaxisymmetric rotational instabilities of the protoneutron star (PNS), or from the recently proposed PNS core g-mode oscillations [1]. Previous estimates of the GW signature of core-collapse supernovae have relied either on Newtonian simulations [2-6] (to some extent approximating GR effects [7, 8]), or GR simulations with simplified analytic (so-called hybrid) EOSs and no neutrino treatment [9-12]. Depending on the rotation strength, the softness of the EOS at subnuclear densities, and the inclusion of GR effects, the collapse dynamics and, accordingly, the GW signatures can differ significantly.

In previous studies, at least three 'types' of dynamics and resulting GW signatures of rotating collapse were identified: type I dynamics is characterized by little influence of centrifugal effects during collapse and bounce. Type I models undergo core bounce governed by the stiffening of the nuclear EOS at nuclear density and 'ring down' quickly into postbounce equilibrium. Their waveforms exhibit one pronounced large (negative) spike at bounce and then show a gradually damped ring-down wave signal at early postbounce times. Type II models, on the other hand, are rotation dominated and undergo core bounce at densities below (or slightly above) nuclear matter density under the strong influence of centrifugal forces. Their dynamics generally exhibits multiple harmonic-oscillator-like damped slow cycles of coherent bounce-re-expansion-collapse (multiple bounces), which is reflected in the waveform by distinct signal peaks associated with every bounce. Type III dynamics is characterized by fast collapse (owing to very efficient electron capture, for instance, in accretion-induced collapse or large artificial initial pressure reduction in polytropic core-collapse models), extremely small masses of the homologously collapsing inner core and low-amplitude GW emission.

In this paper we present new results from GR simulations, focusing on the rotating collapse, bounce and early postbounce phases. As a first stage we perform 2D (axisymmetric) and 3D calculations with polytropic initial models and a hybrid EOS in order to compare results obtained in the conformally-flat approximation to GR (CFC; conformal-flatness condition $[13,14])$ with results from full Cauchy free evolutions in $3+1$ GR. The second stage encompasses the first ever multi-D simulations of rotating stellar iron core collapse in GR with presupernova models from stellar evolution calculations, a finite-temperature nuclear EOS, and a simple, but effective treatment of electron capture and neutrino radiation effects during collapse. In this way, we obtain the most accurate estimates of the GW signature of rotating stellar core collapse in full GR to date.

This paper is organized as follows. In section 2, we delineate the methods and numerical tools employed and present the initial model data that we utilize in our calculations. In section 3, we present the numerical results of our calculations, focusing on the CFC-fullGR comparison in section 3.1, on the GW signature of axisymmetric rotating core collapse with microphysics in section 3.2 and on the development of nonaxisymmetric structures and dynamics in section 3.3. In section 4 , we summarize our results and discuss the detectability of the GWs from our models. 


\section{Methods and initial model data}

We base this work on two independent numerical codes: the CoCoNuT code of Dimmelmeier et al $[9,15,16]$ which treats GR in the conformally-flat approximation and which we employ in 2D (axisymmetric) mode and CACTUs/CARPET/Whisky (CCW) [17-20] for $3+1$ full free Cauchy evolution GR calculations. CCW uses mesh-refined Cartesian grids while CoCoNuT operates in spherical coordinates. Both codes employ the same formulation of GR hydrodynamics which is outlined in section 2.1. Code details and the different methods for time-updating the GR curvature fields are discussed in section 2.2 for $\mathrm{CCW}$ and in section 2.3 for CoCoNuT. In section 2.4, we discuss the EOSs that we utilize, while section 2.5 deals with the deleptonization and neutrino pressure treatment that we implement in our codes. In section 2.6 we introduce the initial model data used for our calculations, and in section 2.7 we discuss the GW extraction method.

\subsection{GR hydrodynamics}

We adopt the ADM $3+1$ foliation of spacetime [21]. All equations assume $c=G=1$. In the following, Latin indices run from 1 to 3 while Greek ones run from 0 to 3 . We adhere to abstract index notation. $g_{\mu \nu}$ is the 4-metric and $\gamma_{i j}$ is the 3 -metric.

The hydrodynamic evolution of a perfect fluid in GR with 4-velocity $u^{\mu}$, rest-mass current $J^{\mu}=\rho u^{\mu}$, where $\rho$ is the rest-mass density, and stress-energy tensor $T^{\mu \nu}=\rho h u^{\mu} u^{\nu}+P g^{\mu \nu}$ is determined by a system of local conservation equations,

$$
\nabla_{\mu} J^{\mu}=0, \quad \nabla_{\mu} T^{\mu \nu}=0,
$$

where $\nabla_{\mu}$ denotes the covariant derivative with respect to the 4-metric. The quantity $h=1+\epsilon+P / \rho$ is the specific enthalpy, $P$ is the fluid pressure, and the 3 -velocity is given by $v^{i}=u^{i} /\left(\alpha u^{0}\right)+\beta^{i} / \alpha$, where $\alpha$ is the lapse function and $\beta^{i}$ is the coordinate shift. We define the set of conserved variables as

$$
D=\rho W, \quad S^{i}=\rho h W^{2} v^{i}, \quad \tau=\rho h W^{2}-P-D .
$$

In the above expressions $W=\alpha u^{0}$ is the Lorentz factor, which satisfies the relation $W=1 / \sqrt{1-v_{i} v^{i}}$.

The local conservation laws (1) are written as a first-order, flux-conservative system of hyperbolic equations [22],

with

$$
\frac{\partial \sqrt{\gamma} \boldsymbol{U}}{\partial t}+\frac{\partial \sqrt{-g} \boldsymbol{F}^{i}}{\partial x^{i}}=\sqrt{-g} \boldsymbol{S}
$$

$$
\begin{aligned}
& \boldsymbol{U}=\left[D, S_{j}, \tau\right] \\
& \boldsymbol{F}^{i}=\left[D \hat{v}^{i}, S_{j} \hat{v}^{i}+\delta_{j}^{i} P, \tau \hat{v}^{i}+P v^{i}\right] \\
& \boldsymbol{S}=\left[0, T^{\mu \nu}\left(\frac{\partial g_{\nu j}}{\partial x^{\mu}}-\Gamma_{\mu \nu}^{\lambda} g_{\lambda j}\right), \alpha\left(T^{\mu 0} \frac{\partial \ln \alpha}{\partial x^{\mu}}-T^{\mu \nu} \Gamma_{\mu \nu}^{0}\right)\right] .
\end{aligned}
$$

Here $\hat{v}^{i}=v^{i}-\beta^{i} / \alpha$, and $g$ and $\gamma$ are the determinants of $g_{\mu \nu}$ and $\gamma_{i j}$, respectively, with $\sqrt{-g}=\alpha \sqrt{\gamma} . \Gamma_{\mu \nu}^{\lambda}$ are the 4-Christoffel symbols.

The above equations are solved in semi-discrete fashion. The spatial discretization is performed by means of a high-resolution shock-capturing (HRSC) scheme employing a second-order accurate finite-volume discretization. We employ the Marquina flux formula for the local Riemann problems and piecewise-parabolic cell interface reconstruction (PPM). For a review of such methods in the GR context, see [23]. The time integration and coupling with curvature are carried out with the method of lines [24] in combination with a 
second-order accurate explicit Runge-Kutta scheme in $\mathrm{CoCoNuT}$ and a second-order accurate explicit iterated Crank-Nicholson [25] integrator in CCW.

\subsection{CActus/CARPet/Whisky}

In York's variant of the ADM 3 + 1 Cauchy-evolution formalism [21], the Einstein equations split into a coupled set of first-order evolution equations for the 3-metric $\gamma_{i j}$ and the extrinsic curvature $K_{i j}$,

$$
\begin{aligned}
& \partial_{t} \gamma_{i j}=-2 \alpha K_{i j}+\nabla_{i} \beta_{j}+\nabla_{j} \beta_{i}, \\
& \begin{aligned}
\partial_{t} K_{i j}=-\nabla_{i} \nabla_{j} \alpha+\alpha\left(R_{i j}+K K_{i j}-2 K_{i k} K_{j}^{k}\right)+\beta^{k} \nabla_{k} K_{i j} \\
\quad+K_{i k} \nabla_{j} \beta^{k}+K_{j k} \nabla_{i} \beta^{k}-8 \pi \alpha\left(S_{i j}-\frac{\gamma_{i j}}{2}\left(S_{k}^{k}-\rho_{\mathrm{ADM}}\right)\right),
\end{aligned}
\end{aligned}
$$

and constraint equations,

$$
\begin{aligned}
& 0=R+K^{2}-K_{i j} K^{i j}-16 \pi \rho_{\mathrm{ADM}}, \\
& 0=\nabla_{i}\left(K^{i j}-\gamma^{i j} K\right)-8 \pi S^{j} .
\end{aligned}
$$

In the above equations, $R_{i j}$ is the 3-Ricci tensor and $R$ is the scalar 3-curvature. The projection of the stress-energy tensor onto the spatial hypersurface is $S_{i j}=\rho h W^{2} v_{i} v_{j}+\gamma_{i j} P$, the ADM energy density is given by $\rho_{\mathrm{ADM}}=\rho h W^{2}-P$ and $S^{j}=\rho h W^{2} v^{i}$ is the momentum density as measured by an Eulerian observer moving orthogonally to the spacelike hypersurfaces.

In CCW we employ the AEI-BSSN code that implements the BSSN recast of the ADM equations in the fashion detailed in [26, 27]. Here, we mention only that the BSSN formalism makes use of a conformal decomposition of the 3-metric, $\tilde{\gamma}_{i j}=\mathrm{e}^{-4 \phi} \gamma_{i j}$, and the trace-free part of the extrinsic curvature, $A_{i j}=K_{i j}-\gamma_{i j} K / 3$, with the conformal factor $\phi$ chosen to satisfy $\mathrm{e}^{4 \phi}=\gamma^{1 / 3}$. In this formulation, in addition to the evolution equations for the conformal 3-metric $\tilde{\gamma}_{i j}$ and the conformal traceless extrinsic curvature $\tilde{A}_{i j}$, there are evolution equations for the conformal factor $\phi$, for the trace of the extrinsic curvature $K$, and for the 'conformal connection functions' $\tilde{\Gamma}^{i} \equiv \partial \tilde{\gamma}^{i j} / \partial x^{j}$.

We employ the common $1+\log$ slicing condition and a $\Gamma$-driver type condition for the shift [27], $\partial / \partial t \beta^{i}=\left(\tilde{\Gamma}^{i}+\Delta t \partial / \partial t \tilde{\Gamma}^{i}\right)$, which is similar to that used in [10].

Mesh refinement in CCW is provided by the CARPET driver [28] which implements full Berger-Oliger mesh refinement [29] with subcycling in time. In the calculations presented here we employ box-in-box refinement hierarchies with a maximum of nine levels of refinement and a factor of 2 increase in resolution from one level to the next. We run CARPET in progressive mesh refinement mode and activate predefined refinement levels based on a density criterion as the collapse proceeds [20]. The grids extend to a maximum outer diagonal radius of $\sim 5000 \mathrm{~km}$ and the finest grid has a linear zone size of $\sim 350 \mathrm{~m}$.

\subsection{CoCoNuT}

In the CoCoNuT code we employ the CFC approximation to GR introduced by Isenberg [13] and first used in a pseudo-evolutionary context by Wilson et al [14]. Details on the CFC GR equations and implementation specifics can be found in $[15,16]$. Here we mention only the salient features of CFC in which the ADM spatial 3-metric is replaced by the conformallyflat 3-metric, $\gamma_{i j}=\phi^{4} \hat{\gamma}_{i j}$, where $\hat{\gamma}_{i j}$ is the flat-space metric. Hence $\hat{\gamma}_{i j}=\delta_{i j}$ in Cartesian coordinates and $\hat{\gamma}_{i j}=\operatorname{diag}\left(1, r^{2}, r^{2} \sin \theta\right)$ in spherical coordinates. Here $\phi$ is the conformal factor. 
In the CFC approximation the ADM equations (3)-(6) reduce to a set of elliptic equations for $\phi, \alpha$ and $\beta^{i}$, if additionally maximal slicing is assumed: $K=0$. The extrinsic curvature becomes a function of these variables and the shift is to leading order (for matter-dominated spacetimes) identical to the minimal distortion condition [21]. The CFC ADM equations do not contain explicit time derivatives, and thus the CFC spacetime fields are evaluated in a fully constrained approach and at each timestep anew.

Imposing CFC in a spherically symmetric spacetime is equivalent to solving the exact Einstein equations. For nonspherically symmetric configurations the CFC approximation may be roughly regarded as full GR minus the dynamical degrees of freedom of the gravitational field that correspond to the GW content [30]. However, even spacetimes that do not contain GWs can be non-conformally flat. Prime examples are the spacetime of a Kerr black hole [31] or rotating fluids in equilibrium. For rapidly rotating stationary neutron stars the deviation of certain metric components from conformal flatness has been shown to reach up to $\sim 5 \%$ in extreme cases [50], while the fundamental oscillation frequencies of such models typically deviate even less from the corresponding values obtained in full GR simulations [33].

Due to its fully constrained nature, the CFC approximation permits a straightforward and numerically more robust implementation of the metric equations in coordinate systems containing coordinate singularities (e.g., spherical polar coordinates in axisymmetry and 3D) compared to the Cauchy free evolution scheme employed in CCW.

In CoCoNuT we use Eulerian spherical coordinates and assume axisymmetry for the corecollapse simulations discussed here. The computational grids consist of 250 logarithmicallyspaced and centrally-condensed radial zones with a central resolution of $250 \mathrm{~m}$ and 45 equidistant angular zones covering $90^{\circ}$.

\subsection{Equations of state}

For calculations employing polytropes in rotational equilibrium, we utilize the hybrid polytropic-ideal-fluid EOS first introduced by [34]. It was discussed and used in many previous studies involving polytropic iron core models (see, e.g., $[3,9,10,15,16])$. At densities below nuclear matter density $\rho_{\text {nuc }}=2 \times 10^{14} \mathrm{~g} \mathrm{~cm}^{-3}$, we choose a polytropic $\Gamma=\Gamma_{1} \lesssim 4 / 3$ from the set $\{1.325,1.320,1.310,1.300,1.280\}$ corresponding to labels $\{\mathrm{G} 1, \mathrm{G} 2, \mathrm{G} 3, \mathrm{G} 4, \mathrm{G} 5\}$ (see also section 2.6.1). Above $\rho_{\text {nuc }}, \Gamma$ is set to $\Gamma_{2}=2.5$ to mimic the stiffening of the nuclear EOS. The hybrid EOS provides for a smooth transition between the two density regimes. A thermal contribution owing to shock heating after core bounce is modelled via a $\Gamma$-law ideal gas EOS with $\Gamma_{\text {th }}$ set to 1.5 .

In our more realistic model calculations we employ the tabulated finite-temperature nuclear EOS by Shen et al [35] (Shen EOS) in the variant of Marek et al [36] which includes baryonic, electronic and photonic pressure components. The Shen EOS returns the fluid pressure (and additional thermodynamic quantities) as a function of $\left(\rho, T, Y_{e}\right)$, where $T$ is the temperature and $Y_{e}$ is the electron number fraction per baryon for which we additionally solve the advection equation

$$
\frac{1}{\sqrt{-g}}\left(\frac{\partial\left(\sqrt{\gamma} D Y_{e}\right)}{\partial t}+\frac{\partial\left(\sqrt{-g} D Y_{e}\left(\alpha v^{i}-\beta^{i}\right)\right)}{\partial x^{i}}\right)=S_{Y_{e}},
$$

where $S_{Y_{e}}$ is a sink term owing to electron capture as discussed in section 2.5. Since the codes operate with the specific internal energy $\epsilon$, we determine the corresponding temperature $T$ iteratively with a Newton-Raphson scheme and the EOS table. All interpolations are carried out in tri-linear fashion and the table is sufficiently densely spaced to lead to an artificial entropy increase in adiabatic collapse by not more than $\sim 2 \%$. 


\subsection{Deleptonization and neutrino pressure}

Electron capture during collapse reduces $Y_{e}$ (i.e. 'deleptonizes' the collapsing core) and consequently decreases the size of the homologously collapsing inner core that roughly has a $Y_{e}^{2}$ dependence (see, e.g., [37]). The material of the inner core is in sonic contact and determines the dynamics and the GW signal at core bounce and in the early postbounce phases. Hence, it is important to include deleptonization during collapse. Since multi-D GR radiation-hydrodynamics calculations are still computationally infeasible, in the simulations using the Shen EOS we employ a recently proposed scheme [38] in which deleptonization is parametrized based on data from detailed 1D radiation-hydrodynamics calculations. For this we use the results obtained with the VERTEX code and the standard set of electron capture rates on free protons and protons bound in heavy nuclei [39]. Following [38], deleptonization is turned off at core bounce (defined as the point in simulation time when the specific entropy per baryon $s$ at the edge of the inner core reaches $3 k_{\mathrm{B}}$ ). After core bounce $Y_{e}$ is passively advected, but the postbounce deleptonization of the PNS is not tracked.

Neutrino pressure is included only in the neutrino optically-thick regime (at $\rho \gtrsim$ $\rho_{\text {trapping }}=2 \times 10^{12} \mathrm{~g} \mathrm{~cm}^{-3}$ ) where we treat the neutrino pressure contribution $P_{v}$ as an ideal Fermi gas as discussed in [38], and include the radiation stress via source terms in the momentum and energy equations. Concretely, we add in fully-coupled method-of-lines fashion $-\alpha \sqrt{\gamma} \partial P_{\nu} / \partial x^{i}$ and $-\alpha \sqrt{\gamma} v^{i} \partial P_{\nu} / \partial x^{i}$ as source terms to the right-hand side of the momentum and energy equations, respectively. A detailed discussion of our implementation, identical in both CoCoNuT and CCW, can be found in [20].

\subsection{Initial models}

2.6.1. Polytropes in rotational equilibrium. For the comparison between CFC (CoCoNuT) and full GR $(\mathrm{CCW})$ we employ $\Gamma=4 / 3$ (i.e. $n=3$ ) polytropes in rotational equilibrium that are obtained with the relativistic extension of Hachisu's self-consistent field method [41]. The polytropes are set up with the rotation law discussed in $[3,15]$ and are parametrized via the differential rotation parameter $A$ and the initial ratio $\beta_{\mathrm{i}}$ of rotational kinetic energy $T$ to gravitational binding energy $|W|$. We employ the model names of [3] that relate $\{\mathrm{A} 1, \mathrm{~A} 2, \mathrm{~A} 3, \mathrm{~A} 4\}$ with $\{A=50000 \mathrm{~km}, A=1000 \mathrm{~km}, A=500 \mathrm{~km}, A=100 \mathrm{~km}\}$ and $\{\mathrm{B} 1, \mathrm{~B} 2, \mathrm{~B} 3, \mathrm{~B} 4, \mathrm{~B} 5\}$ with $\left\{\beta_{\mathrm{i}}=0.25 \%, \beta_{\mathrm{i}}=0.50 \%, \beta_{\mathrm{i}}=0.90 \%, \beta_{\mathrm{i}}=1.80 \%, \beta_{\mathrm{i}}=\right.$ $4.00 \%$. Note that the degree of differential rotation decreases with increasing $A$. The model names are constructed based on the values of $A$ and $\beta_{\mathrm{i}}$ employed and on the value of the polytropic index $\Gamma_{1}$ used in the subnuclear regime (see section 2.4). Hence, model A1B3G5, for example, has $A=50000 \mathrm{~km}, \beta_{\mathrm{i}}=0.90 \%$ and is evolved with $\Gamma_{1}=1.280$.

For the comparison study we choose models A1B3G3 (yielding type I dynamics and waveform), A3B3G2 (type I/II transitional), A2B4G1 (type II) and A1B3G5 (type III) as a representative subset of the models considered by [9].

2.6.2. Presupernova models from stellar evolutionary calculations. All presupernova stellar models available to date are end products of Newtonian spherically symmetric (1D) stellar evolutionary calculations from hydrogen burning on the main sequence to the onset of core collapse by photo-dissociation of heavy nuclei and electron capture (see, e.g., [42]). Recently, the first presupernova models that include rotation in an approximate $1 \mathrm{D}$ fashion have become available [43, 44]. Here, we employ a solar-metallicity $20 M_{\odot}$ (at zero-age main sequence) model of [42] (in the following, model s20) and set it into rotation according to the rotation law specified in [3] and with the same rotation nomenclature employed for the above polytropes (such as A2B4). In addition, we perform calculations with the 'rotating' presupernova model 
Table 1. Summary of the models including microphysics. $\rho_{\mathrm{b}}$ is the density at bounce, the maximum characteristic GW strain [48] $h_{\mathrm{char} \text { max }}$ is at a distance of $10 \mathrm{kpc}$, and $E_{\mathrm{gw}}$ is the energy emitted in GWs (see, e.g., [16]). Models s20A2B4 and E20A are evolved to $\sim 90 \mathrm{~ms}$ and $\sim 70 \mathrm{~ms}$ after bounce, respectively. The table columns with model names that have the subscript 'axi' include only the GW emission from their axisymmetric phases up to $\sim 10-20 \mathrm{~ms}$ after bounce.

\begin{tabular}{lcccccc}
\hline Model & $A\left(10^{8} \mathrm{~cm}\right)$ & $\beta_{\mathrm{i}}(\%)$ & $\beta_{\mathrm{b}}(\%)$ & $\rho_{\mathrm{b}}\left(\frac{10^{14} \mathrm{~g}}{\mathrm{~cm}^{3}}\right)$ & $h_{\text {char, max }}\left(10^{-21}\right)$ & $E_{\mathrm{gw}}\left(10^{-9} M_{\odot} c^{2}\right)$ \\
\hline s20A1B1 & 50.0 & 0.25 & 0.90 & 3.29 & 1.46 & 0.6 \\
s20A1B5 & 50.0 & 4.00 & 10.52 & 2.90 & 9.68 & 26.9 \\
s20A2B2 & 1.0 & 0.50 & 6.72 & 3.07 & 8.77 & 22.0 \\
s20A3B3 & 0.5 & 0.90 & 16.57 & 2.33 & 4.58 & 12.4 \\
s20A2B4 & 1.0 & 1.80 & 16.33 & 2.35 & 4.28 & 9.4 \\
s20A2B4 & & & & & 64.23 & 169.0 \\
E20A & & 0.37 & 11.31 & 2.79 & 12.18 & 36.9 \\
E20A & - & & & 24.23 & 75.4 \\
\hline
\end{tabular}

E20A of [43], which we map onto our computational grids under the assumption of constant rotation on cylindrical shells of constant coordinate radius. We point out that due to their $1 \mathrm{D}$ nature, none of the considered models are in rotational equilibrium. This should not limit the quality of our results, since the collapse proceeds slowly on a timescale of more than $100 \mathrm{~ms}$ and, hence, the star has sufficient time for the adjustment to the appropriate angular stratifications for its rate of rotation $[2,3]$. Initial curvature data in $\mathrm{CCW}$ are obtained via the Newtonian metric approximation [45] and in CoCoNuT via the CFC ADM equations.

In this study, we focus on the collapse of massive presupernova iron cores with at most moderate differential rotation and maximum precollapse rotation rates that lead to PNSs that are likely spinning too fast to yield cold NS spin periods in agreement with observationally inferred injection periods of young pulsars into the $P / \dot{P}$ diagram $[44,46]$. However, they may be relevant in the collapsar-type gamma-ray burst scenario [46, 47].

In table 1, we summarize the parameters of the models including microphysics calculated in this work.

\subsection{Gravitational wave extraction}

We employ the Newtonian quadrupole formula in the first-moment of momentum density formulation as discussed in [16] to extract the GWs generated by aspherical accelerated fluid motions. We point out that although the quadrupole formula is not gauge invariant and is only valid in the Newtonian slow-motion limit, it yields results that agree very well in phase and to $\sim 10-20 \%$ in amplitude with more sophisticated methods [40].

\section{Results}

\subsection{Simple hybrid EOS: comparison between CFC and full GR}

In the following, we compare the time evolution of the maximum rest-mass density $\rho_{\max }$ and the GW signals of a set of models obtained in CFC with CoCoNuT and in full GR with CCW. When carrying out such a comparison of generally coordinate-dependent quantities, it is important to understand in what way differences in the gauge conditions might affect the comparison. Since any spherically symmetric metric can be expressed as conformally-related to the flat-space metric, CFC is an exact representation of full GR in spherical symmetry (see section 2.3). Hence, we compare the CFC gauge with the gauge conditions employed in $\mathrm{CCW}$ in a spherically symmetric calculation and find that both slicing and spatial coordinate 

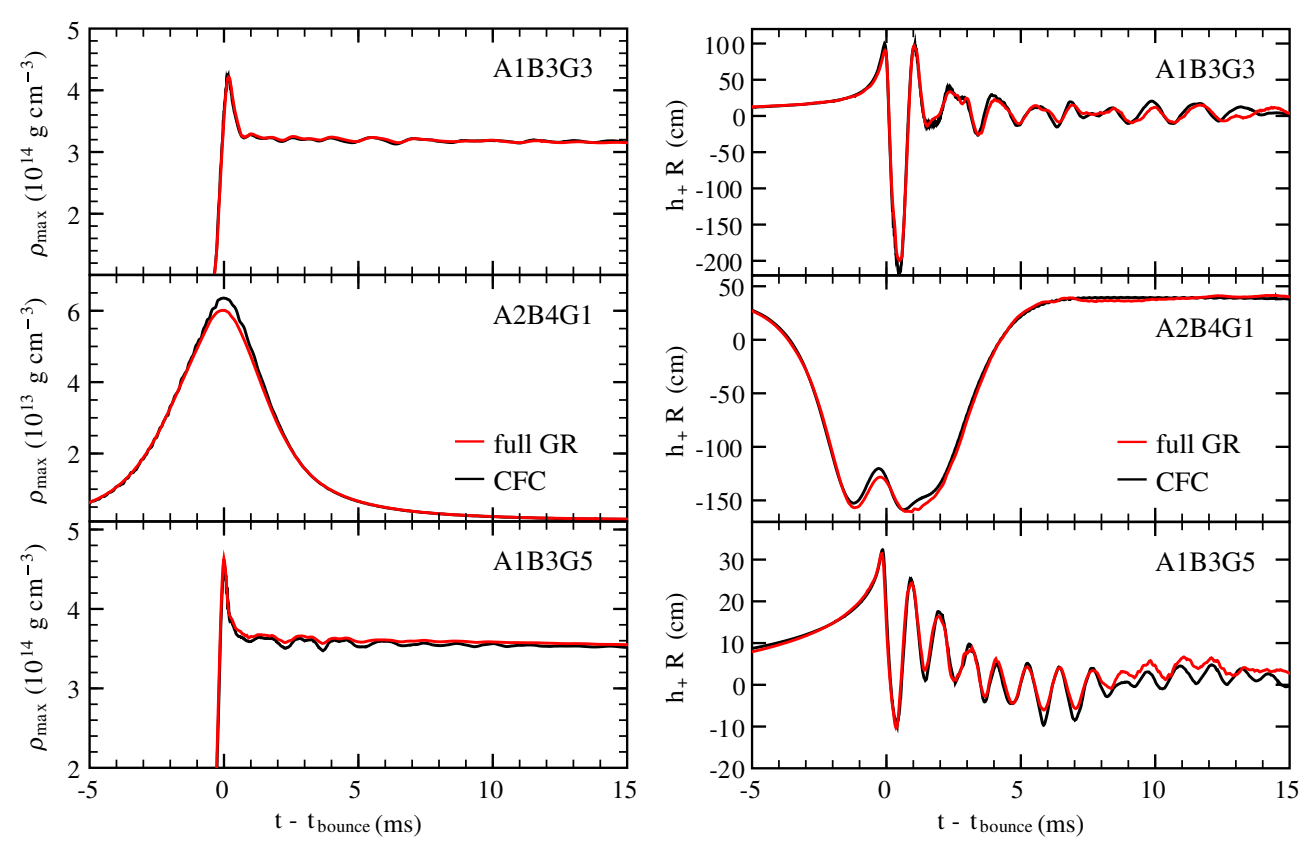

Figure 1. Comparison of the time evolution of $\rho_{\max }$ and of $\mathrm{GW}$ signals (in units of $\mathrm{cm}$; rescaled by observer distance $R$ ) obtained in full GR (red curves) and CFC (black curves) for models A1B3G3 (type I), A2B4G1 (type II) and A1B3G5 (type III).

conditions match very well, independent of the assumption of spherical symmetry in the case of matter-dominated spacetimes [20]. It is hence safe to employ coordinate-dependent quantities for the comparison of results from CoCoNuT and CCW in the stellar core collapse scenario, provided the gauge conditions presented in sections 2.2 and 2.3 are used.

In figure 1, we compare time evolutions of $\rho_{\max }$ and GW signals obtained in CFC and full GR for models A1B3G3 (type I), A2B4G1 (type II) and A1B3G5 (type III). The CFC and full GR results agree very well for all models, modulo small differences varying from model to model in the degree to which small features in $\rho_{\max }$ and in the waveforms are resolved. These differences are most likely due to (i) differences in the numerical implementations, (ii) different artificial numerical damping due to the different choices of computational coordinates (spherical versus Cartesian) and (iii) slight under-resolution of the dynamics by either $\mathrm{CCW}$ or CoCoNuT. Importantly, our results show no signs of errors in the dynamics that could be caused by the CFC approximation of GR in core collapse. This finding is in agreement with previous results [10,12].

In order to more quantitatively assess how far a given core-collapse spacetime deviates from conformal flatness, we calculate the Cotton-York tensor [6, 30, 49]

$$
Y^{i j}=\epsilon^{i l m} \nabla_{l}\left(R_{m}^{j}-\frac{1}{4} \delta_{m}^{j} R\right),
$$

which vanishes on conformally-flat slices. Following [49], we use its matrix norm normalized by the covariant derivative of the 3-Ricci tensor, $H=\left\|Y_{i j}\right\| /\left(\nabla_{i} R_{j k} \nabla^{i} R^{j k}\right)$, to construct the rest-mass density weighted integral quantity

$$
\langle H\rangle_{\rho}=\frac{\int \mathrm{d}^{3} x H \sqrt{\gamma} \rho W}{\int \mathrm{d}^{3} x \sqrt{\gamma} \rho W}
$$

as a measure of the deviation from conformal flatness. We perform test calculations with the Cotton-York analysis for models A1B3G3 and A3B3G2 and plot the time evolution of $\langle H\rangle_{\rho}$ 


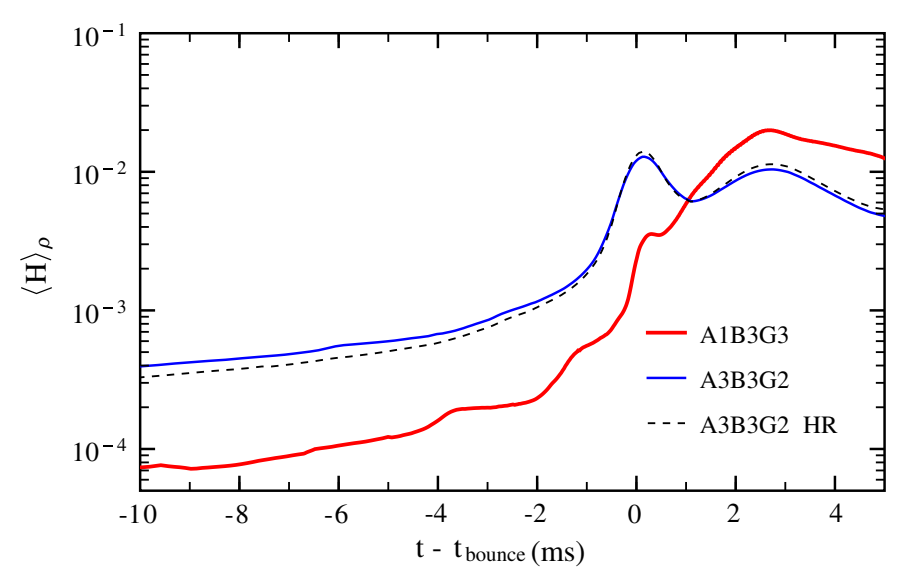

Figure 2. Density-weighted volume integral of the matrix norm of the Cotton-York tensor (8) and (9) at times shortly before and after core bounce in models A1B3G3 and A3B3G2. In addition, we plot $\langle H\rangle_{\rho}$ in a calculation of model A3B3G2 with a resolution increased overall by $20 \%$ (labelled HR). The numerics are reasonably well converged. Note that the rotationally more flattened type I/II transitional model A3B3G2 achieves higher prebounce $\langle H\rangle_{\rho}$, but, owing to smaller compactness, lower postbounce $\langle H\rangle_{\rho}$ than the type I model A1B3G3.

in figure 2. If one can (in a quantitative way) trust the normalization proposed by [49], then the core-collapse spacetimes studied here deviate from conformal flatness by not more than a few per cent at and shortly after core bounce (which is in agreement with previous work $[12,50]$. The situation is very likely to be different at later postbounce times (not considered here) when the PNS has accreted a significant amount of mass, cooled and shrunk, and is thus more compact.

\subsection{Calculations with presupernova models and microphysics: axisymmetric collapse dynamics and waveforms}

In this section, we present new results for the GW signature of the axisymmetric rotating collapse, core bounce and early postbounce epoch of core-collapse supernovae. These results are obtained with CCW, the Shen EOS, deleptonization during collapse and neutrino pressure effects, as delineated in section 2. For all models, counterpart calculations are carried out with CoCoNuT in axisymmetry for verification. The results compare as well as (or better than) those discussed for the models with simple hybrid EOS in section 3.1 (see also [19]).

A first and important result of our study is that all models considered and listed in table 1 remain essentially axisymmetric during collapse, bounce and the immediate postbounce phase (most models are evolved to $\sim 10-20 \mathrm{~ms}$ after bounce). The highest $\beta$ reached at core bounce is $\sim 16.6 \%$. This is much below the threshold value of $\beta \sim 27 \%$ for the high- $T /|W|$ dynamical rotational bar-mode instability and only slightly above the threshold for secular (viscosity and/or GW-reaction driven) rotational instability that would develop on much longer timescales than considered here. Furthermore, and in agreement with previous results $[5,46,51]$, our calculations indicate a natural centrifugal barrier that limits the maximum value of $\beta$ that can be reached during core collapse when a microphysical EOS and presupernova models from stellar evolution calculations are employed.

In figure 3, we present gravitational waveforms of models with varying initial degree of differential rotation $A$ and rotation rate $\beta_{\mathrm{i}}$. This figure demonstrates that, largely independent of the initial rotational configuration in the parameter space considered here, the GW signal of 


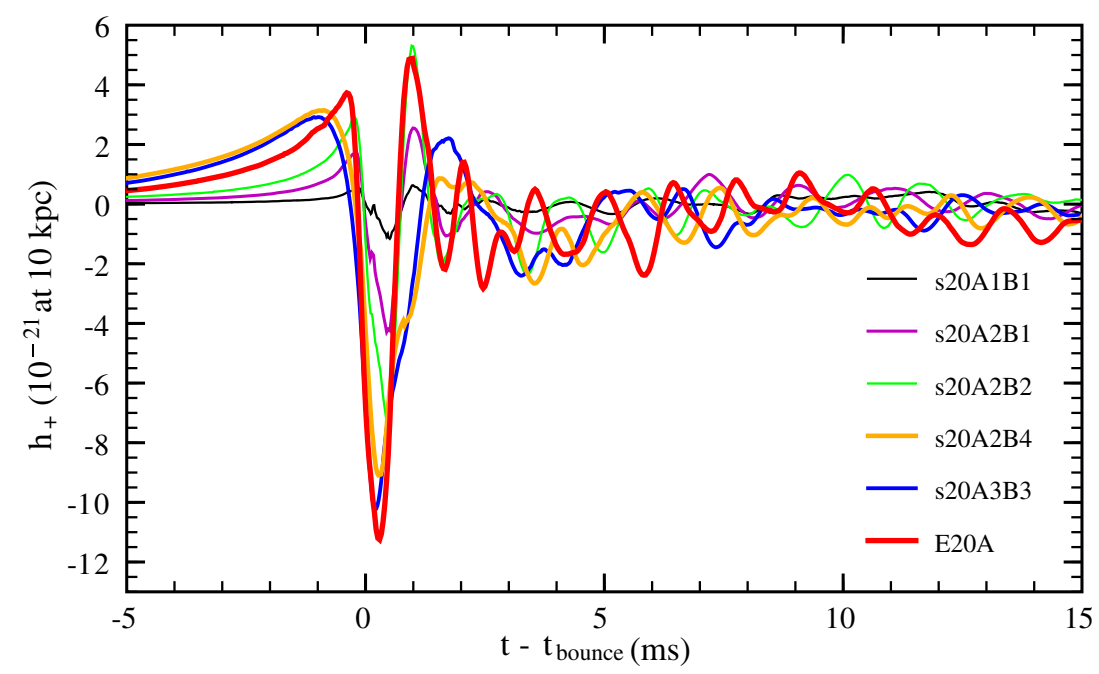

Figure 3. GW strain $h_{+}$along the equator for all model calculations employing presupernova models from stellar evolutionary studies, the Shen EOS, deleptonization during collapse, and neutrino pressure in the neutrino optically-thick regime. Note the generic shape of the waveforms that is largely independent of precollapse rotation rate and degree of differential rotation in the parameter space chosen here.

core bounce in rotating collapse has a generic shape: a slow signal increase in the prebounce phase, a large negative amplitude at core bounce when the motion of the quasi-homologously collapsing inner core is reversed, followed by a ring-down. This is a clear type I signature corresponding to a baryonic pressure-dominated bounce. All our microphysical models undergo core bounce dominated by the stiffening of the EOS at nuclear density, and most of them radiate the largest fraction of their GW energy in a relatively narrow band (with $\delta f \sim 50 \mathrm{~Hz}$ ) centred in the range between about 650 and $800 \mathrm{~Hz}$. With increasing influence of centrifugal effects, the peaks of the GW energy spectra shift to lower frequencies, reaching $\sim 300 \mathrm{~Hz}$ for the axisymmetric emission in model s20A2B4.

The above is in stark contrast to the studies using the hybrid EOS [3, 9-11] (see also section 3.1), where initial models with rotation rates in the range investigated here develop sufficient centrifugal support during contraction to stop the collapse at subnuclear densities, resulting in several consecutive centrifugal bounces separated by phases of coherent reexpansion of the inner core. While in GR such models exhibiting a multiple centrifugal bounce and the corresponding type II GW signals are only rarer compared to Newtonian gravity [9] if a hybrid EOS is used (which is identical to a polytrope before bounce), we do not observe any such model in our microphysical models. An evident example is model s20A2B4: in previous studies without microphysics, the corresponding model with identical initial rotation parameters and a subnuclear $\Gamma_{1}$ close to $4 / 3$ like A2B4G1 with $\Gamma_{1}=1.325$ (or A2B4G2 with $\Gamma_{1}=1.320$, not presented here) showed clear type II behaviour in both Newtonian and GR calculations [3, 9].

The suppression of the multiple centrifugal bounce scenario is due to two physical effects. On the one hand, GR results in a stronger gravitational pull as compared to a purely Newtonian treatment, thus forming a smaller, more compact PNS with higher maximum density. This effectively stronger gravity in GR severely limits the region in rotational parameter space which permits multiple centrifugal bounces [9]. Additionally, and even more importantly, in contrast to the simple hybrid EOS, in our case the mass and dynamics of the inner core 
(which is most important for the GW emission) is not merely determined by the adiabatic index $\Gamma=\mathrm{d} \ln P / \mathrm{d} \ln \rho$ (at constant entropy) of the EOS, but also by deleptonization during collapse. This leads to considerably smaller inner cores with less angular momentum and weaker pressure support [52], which again suppresses multiple centrifugal bounces in a very efficacious way. However, even when including the effects of deleptonization, the mass of the inner core is still substantially larger than when a hybrid EOS with $\Gamma_{1} \lesssim 1.300$ at subnuclear densities is used. This explains why we do not observe any type III waveforms in the microphysical models, as these require an extremely small mass of the inner core [3,9].

An instructive example for the impact of deleptonization and GR on the collapse dynamics is the type II model D from the Newtonian study of Mönchmeyer and Müller [2], where a microphysical finite-temperature EOS and an approximate deleptonization scheme were employed (including only electron capture on protons and neglecting captures on heavy nuclei which leads to larger $Y_{e}$ and inner core masses at core bounce than observed in our models). When abandoning the restrictions of the deleptonization scheme or gravity in that work by using a more modern prescription for electron capture [39] or including GR effects (and certainly by combining both), we now find that their model D exhibits unambigous pressuredominated collapse dynamics with a type I GW signal. A detailed analysis of the interplay and quantitative influence of the above two effects responsible for the elimination of multiple centrifugal bounces in the rotating stellar core-collapse scenario is discussed in [20] and will be presented in a future publication [53].

\subsection{Calculations with presupernova models and microphysics: nonaxisymmetric dynamics}

In recent studies dynamical rotational instabilities of $m=2$ and/or $m=1$ character were found in equilibrium polytropic stellar models (in Newtonian gravity and GR; see, e.g., $[10,55,56,58]$, and references therein) and in simplified postbounce PNS models [59] at low $\beta$ in the range of $\sim 1-15 \%$. In contrast to the classical high- $T /|W|$ instability, this new kind of dynamical instability appears to be related to resonant amplification of azimuthal fluid modes at corotation points, where the pattern speed of the mode $\sigma_{\mathrm{p}}=\sigma / m$ (where $\sigma$ is the mode frequency) coincides with the local angular velocity $[55,56,59,60]$.

In the light of the previous results on low- $T /|W|$ corotation instabilities, we continue the postbounce evolution of models E20A and s20A2B4 in order to investigate the development of nonaxisymmetric structure in the PNS and postshock regions of our physically more realistic models. Models E20A and s20A2B4 are both relatively quickly spinning and bracket a range of postbounce $\beta \mathrm{s}$ of $\sim 9-13 \%$. We perform an analysis of azimuthal density modes $\propto \mathrm{e}^{\mathrm{i} m \varphi}$ in the equatorial plane by computing the complex Fourier amplitudes $C_{m}=\frac{1}{2 \pi} \int_{0}^{2 \pi} \rho(\varpi, \varphi, z=0) \mathrm{e}^{\mathrm{i} m \varphi} \mathrm{d} \varphi$ on rings of constant coordinate radius. In the top panels of figure 4 we display the normalized mode amplitudes $A_{m}=\left|C_{m}\right| / C_{0}$ extracted in the two models at $15 \mathrm{~km}$ radius. Without adding artificial seed perturbations to our calculations, discretization errors and the finite accuracy of the Newton-Raphson scheme employed in the EOS routines trigger $m=\{1,2,3\}$ modes, which rise to a level of $\sim 10^{-5}$ during the collapse phases lasting $\sim 170 \mathrm{~ms}$ and $\sim 200 \mathrm{~ms}$ in models s20A2B4 and E20A, respectively.

In both models, the $m=1$ mode grows fastest and with a dynamical rate at core bounce and surpasses the ambient Cartesian $m=4$ grid mode at $\sim 20-30 \mathrm{~ms}$ after bounce, reaching maximum normalized amplitudes of up to $10^{-1} \cdot m=\{2,3\}$ modes grow as well, but at a lower rate, and reach smaller amplitudes than the $m=1$ mode. Note that both models remain dynamically stable to the $m=4$ grid mode.

In the lower halves of the two panels of figure 4 we plot the GW strains $h_{+}$and $h_{\times}$as seen by an observer located on the polar axis at $10 \mathrm{kpc}$ distance. The rotational symmetry 

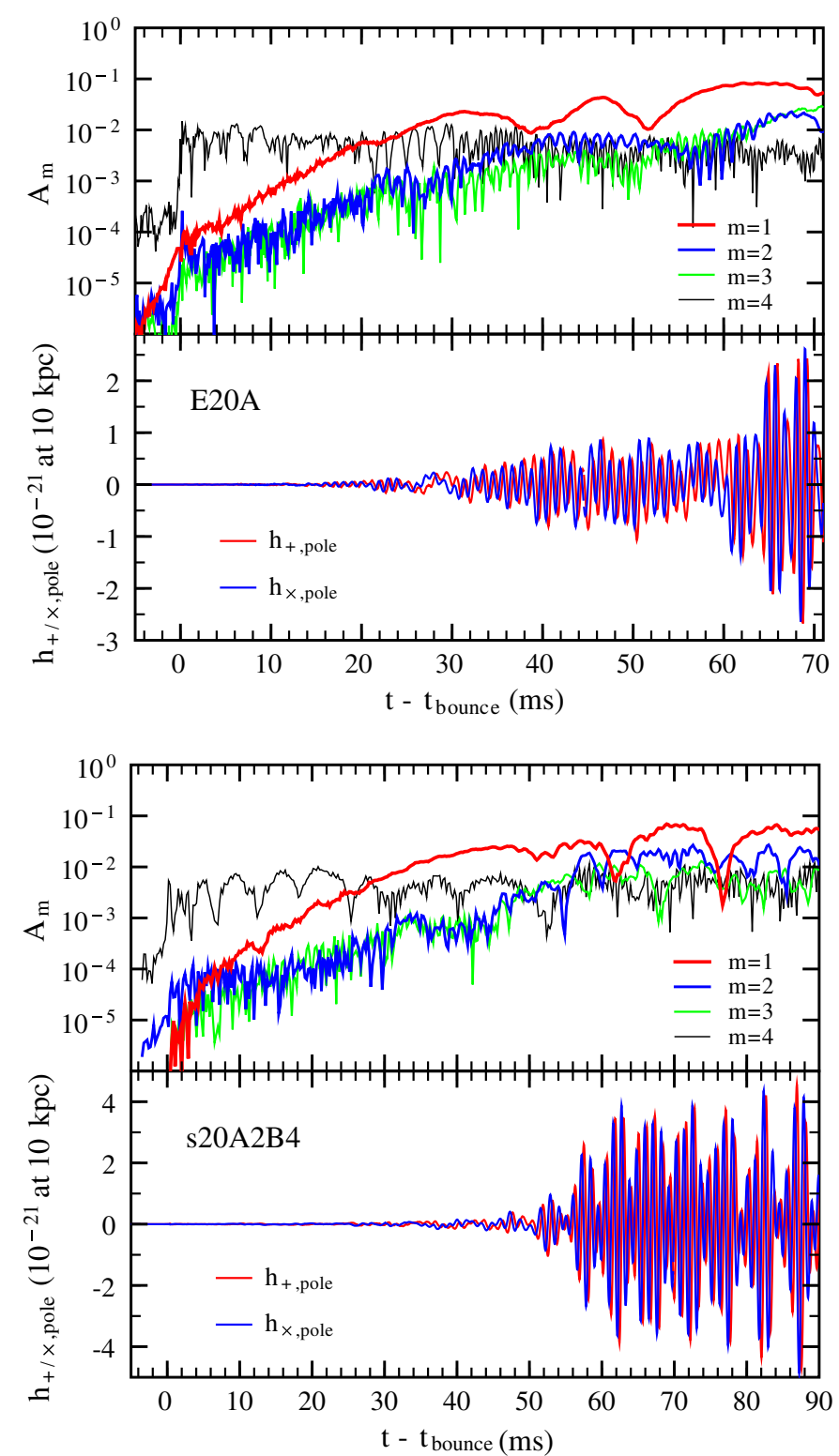

Figure 4. Normalized mode amplitudes $A_{m}$ in the equatorial plane extracted at a radius of $15 \mathrm{~km}$ at postbounce times (upper half of the panels), and GW strains $h_{+}$and $h_{\times}$along the poles (lower half of the panels) at postbounce times in models E20A (upper panel) and s20A2B4 (lower panel). The times are given relative to the time of core bounce in each model.

prevailing at bounce and early postbounce times is apparent from the fact that $h_{+}$as well as $h_{\times}$at the pole is essentially zero until the models develop considerable nonaxisymmetry at $\sim 30 \mathrm{~ms}$ (in E20A) and $\sim 50 \mathrm{~ms}$ (in s20A2B4) after bounce. The GW emission along the polar axis is entirely due to the quadrupole components of the nonaxisymmetric dynamics and, hence, the time at which the GW signals become strong is correlated with the evolution of the $m=2$ mode amplitude. This fact is most clearly portrayed by model s20A2B4 whose GW emission sets in at the time when the $m=2$ mode amplitude crosses that of the background 


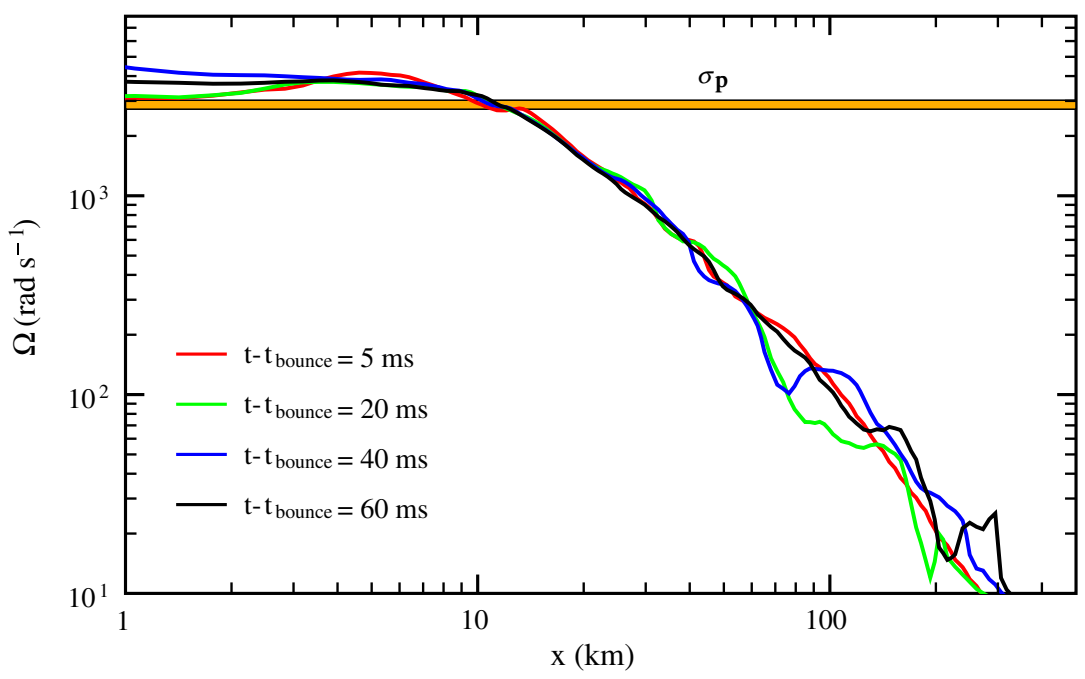

Figure 5. Angular velocity profiles along the positive $x$-axis in the equatorial plane in model E20A at various postbounce times. The pattern speed of the $m=1$ mode is marked with a bar. It is in corotation with the PNS at $\sim 10-15 \mathrm{~km}$. Note that out to $\sim 10 \mathrm{~km}$ the PNS core is in approximate solid body rotation. This is a consequence of the quasi-homologous collapse of the inner iron core [46]. Model s20A2B4 (not shown here) exhibits qualitatively and quantitatively similar features and its $m=1$ mode is in corotation in the same equatorial radial interval.

$m=4$ mode. The GW emission is in a very narrow frequency band (with $\delta f \sim 30 \mathrm{~Hz}$ ). The energy spectra peak at $\sim 928 \mathrm{~Hz}$ for model E20A and at $\sim 918 \mathrm{~Hz}$ for model s20A2B4. In remarkable agreement with expectations for a simple spinning-bar model, $h_{+}$and $h_{\times}$in both models oscillate at the same frequency and are phase-shifted by a quarter cycle.

By analysing the nonaxisymmetric mode structure in both models we find (i) that the pattern speeds of the $m=\{2,3\}$ modes agree with that of the $m=1$ mode, indicating nonlinear mode coupling and (ii) that the GW emission occurs at a frequency corresponding to twice the pattern speed of the $m=1$ mode. In figure 5, we compare the $m=1$ pattern speed with equatorial angular velocity profiles in model E20A. The $m=1$ mode is in corotation with the fluid throughout the postbounce phase at the edge of the PNS core at $\sim 10-15 \mathrm{~km}$, where the shear energy stored in differential rotation is large. This is evidence for the corotation low- $T /|W|$ nature of the rotational instability observed here $[55,56,59,60]$.

\section{Summary and conclusions}

The study presented in this paper relies on $3+1$ full GR and $2+1$ CFC-GR calculations of the collapse of rapidly rotating stellar iron cores to PNSs. The $3+1$ calculations are the first of their kind that are carried out in fully self-consistent fashion on mesh-refined Cartesian grids. Our calculations are the first ever multi-D calculations of core collapse in GR that include a finite-temperature nuclear EOS, presupernova models from stellar evolutionary studies, deleptonization during collapse and neutrino pressure effects.

By comparing results from fully GR and CFC collapse calculations we observe no significant deviations that could be attributed to systematic deficiencies of the CFC approximation in the stellar iron core collapse context. By means of the Cotton-York tensor (which vanishes on conformally-flat spacelike slices) we find that the prebounce and early postbounce spacetimes do not deviate from conformal flatness by more than a few per cent. The 


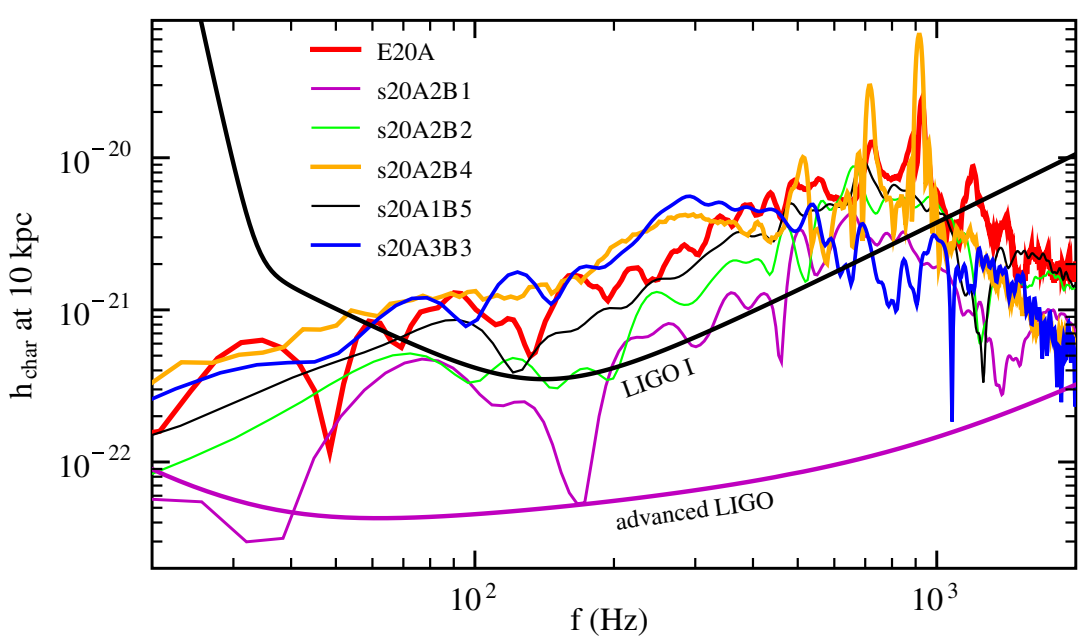

Figure 6. Frequency spectra of the characteristic GW strain $h_{\text {char }}$ of all models with microphysics (scaled to $10 \mathrm{kpc}$ distance) and the initial and advanced LIGO (optimal) rms noise curves [61].

CFC approximation employed in CoCoNuT is, hence, an excellent choice for the modelling of rotating core collapse. In addition, we point out that the capability of CoCoNuT to perform the evolution in spherical coordinates combined with its high computational efficiency in axisymmetry makes it particularly well suited for studying matter-dominated spacetimes in rotational symmetry.

Our results show that the GW signature of the collapse, core bounce and early postbounce phases of the core-collapse supernova evolution is much more generic than previously thought. We find that the dynamics of core bounce is dominated by gravity and microphysics, reducing the relevance of centrifugal support for the wide range of initial rotation rates and degrees of differential rotation considered here. Importantly, for our model set we do not observe rotationally-induced multiple core bounces as proposed by previous studies that did not include a microphysical finite-temperature nuclear EOS and a deleptonization treatment in combination with GR.

All models stay axisymmetric throughout collapse, bounce and the very early postbounce phase, and none of them reach the limit in $T /|W|$ for the classical dynamical MacLaurin-type rotational instability (see, e.g., [54] for a recent study and references therein). However, models s20A2B4 and E20A, which we evolve to later postbounce times, exhibit the dynamical growth of a nonaxisymmetric low- $T /|W|$ corotation-type $m=1$ instability $[55,56,59,60]$. We also observe $m=\{2,3\}$ daughter modes that apparently grow to significant amplitudes via non-linear mode coupling. Strong GWs are emitted by the quadrupole components of the nonaxisymmetric dynamics. While we carried out long-term postbounce evolutions only for models s20A2B4 and E20A, we point out that other models with similar postbounce PNS structures and rotational configurations are likely to experience the same kind of instability. Our results, which remove the limitations of previous studies, demonstrate that the development of nonaxisymmetric structures is neither limited to Newtonian gravity, simple matter models, equilibrium configurations, nor high values of $\beta$ above the classical instability thresholds, but may rather be a phenomenon occurring generically in differentially rotating compact stars.

For an assessment of the detectability of the GW emissions from our models by initial and advanced LIGO detectors, we consider the characteristic GW strain spectra $h_{\text {char }}=R^{-1} \sqrt{2 \pi^{-2} G c^{-3} \mathrm{~d} E_{\mathrm{GW}} / \mathrm{d} f}$ [48] and compare them to the optimal LIGO rms noise curves [61] in figure 6. Considering only the axisymmetric GW burst from core bounce, 
$h_{\text {char }}$ reaches values up to $\sim 10^{-20}$ and has its maximum between 300 and $800 \mathrm{~Hz}$. The GW emission from the nonaxisymmetric dynamics tracked in models E20A and s20A2B4 occurs at lower GW amplitudes than that from core bounce, but over a prolonged period of many tens of milliseconds and in almost monotone fashion centred around $\sim 920-930 \mathrm{~Hz}$. In total emitted energy and in $h_{\text {char }}$ the GW emission from the nonaxisymmetric instability dwarfs that associated with the axisymmetric core bounce and greatly enhances the GW detectability of these models. Importantly, we point out that at the end of our simulations the nonaxisymmetric dynamics in models E20A and s20A2B4 show no sign of decay. They could potentially continue for hundreds of milliseconds until the supernova explosion puts an end to the infusion of high angular momentum material through the stalled shock and sufficient angular momentum is redistributed by the instability to break corotation.

Based on figure 6 we conclude that the GW signal from axisymmetric core bounce of all models investigated here is likely to be detectable by current and future LIGO-class detectors from anywhere in the Milky Way. Models that become nonaxisymmetrically unstable may be detectable out to much larger distances if the instability persists for a sufficiently long time.

We point out that owing to the nature of the approximate deleptonization treatment employed in this study we are unable to capture postbounce neutrino effects and cannot track the sudden drop in electron fraction inside the PNS core associated with the neutrino burst at shock breakout a few milliseconds after bounce. Hence, our treatment of the late-time postbounce evolution is of limited quality, but will be improved in future work.

\section{Acknowledgments}

We wish to thank N Stergioulas for permission to use his initial data solver and M Miller for providing a copy of his Cotton-York routine which was a helpful guide in code development. We furthermore gratefully acknowledge helpful and stimulating discussions with A Burrows, L Dessart, C Meakin, E Müller, S Ou, L Rezzolla, D Shoemaker, E Seidel, N Stergioulas, M Tiglio, J Tohline and S Yoshida. This research was partially supported by the DFG (SFB/Transregio 7 and SFB 375), by the Joint Institute for Nuclear Astrophysics sub-award no 61-5292UA of NFS award no 86-6004791, by the Center for Computation and Technology at Louisiana State University and by the European Network of Theoretical Astroparticle Physics (ENTApP) under contract number RII3-CT-2004-506222 ILIAS/N6. The calculations were performed on the Peyote cluster at the Albert-Einstein-Institut, on the Supermike cluster at Louisiana State University and on the Teragrid machines located at NCSA (computer time grant no AST05022N).

\section{References}

[1] Burrows A et al 2006 Astrophys. J. 640878

[2] Mönchmeyer R et al 1991 Astron. Astrophys. 246417

[3] Zwerger T and Müller E 1997 Astron. Astrophys. 320209

[4] Kotake K, Yamada S and Sato K 2003 Phys. Rev. D 68044023

[5] Ott C D et al 2004 Astrophys. J. 600834

[6] Ott C D et al 2006 Phys. Rev. Lett. 96201102

[7] Müller E et al 2004 Astrophys. J. 603221

[8] Obergaulinger M et al 2006 Astron. Astrophys. 457209

[9] Dimmelmeier H, Font J A and Müller E 2002 Astron. Astrophys. 393523

[10] Shibata M and Sekiguchi Y-I 2004 Phys. Rev. D 69084024

[11] Shibata M and Sekiguchi Y-I 2005 Phys. Rev. D 71024014

[12] Cerdá-Durán P et al 2005 Astron. Astrophys. 4391033

[13] Isenberg J A 1978 University of Maryland Preprint gr-qc/0702113 
[14] Wilson J R, Mathews G J and Marronetti P 1996 Phys. Rev. D 541317

[15] Dimmelmeier H, Font J A and Müller E 2002 Astron. Astrophys. 388917

[16] Dimmelmeier H et al 2005 Phys. Rev. D 71064023

[17] Goodale T et al 2003 Vector and Parallel Processing_VECPAR'2002: 5th Int. Conf. (Lecture Notes in Computer Science vol 2565) ed J M L M Palma (Berlin: Springer) p 197

[18] Baiotti L et al 2005 Phys. Rev. D 71024035

[19] Ott C D et al 2006 Phys. Rev. Lett. submitted Ott C D et al 2006 Preprint astro-ph/0609819

[20] Ott C D 2006 PhD Thesis Universität Potsdam, Potsdam

[21] York J W 1979 Sources of Gravitational Radiation ed L L Smarr (Cambridge: Cambridge University Press) p 83

[22] Banyuls F et al 1997 Astrophys. J. 476221

[23] Font J A 2003 Living Rev. Rel. 64

[24] Hyman J M 1976 Technical Report COO-3077-139, ERDA Mathematics and Computing Laboratory, Courant Institute of Mathematical Sciences, New York University

[25] Teukolsky S 2000 Phys. Rev. D 61087501

[26] Alcubierre M et al 1999 Phys. Rev. D 62044034

[27] Alcubierre M et al 2003 Phys. Rev. D 67084023

[28] Schnetter E, Hawley S H and Hawke I 2004 Class. Quantum Grav. 211465

[29] Berger M J and Oliger J 1984 J. Comput. Phys. 53484

[30] York J W 1971 Phys. Rev. Lett. 261656

[31] Garat A and Price R H 2000 Phys. Rev. D 61124011

[32] Cook G B, Shapiro S L and Teukolsky S A 1996 Phys. Rev. D 535533

[33] Dimmelmeier H, Stergioulas N and Font J-A 2006 Mon. Not. R. Astron. Soc. 3681609

[34] Janka H-T, Zwerger T and Mönchmeyer R 1993 Astron. Astrophys. 268360

[35] Shen H et al 1998 Prog. Theor. Phys. 1001013

[36] Marek A et al 2005 Astron. Astrophys. 443201

[37] Martínez-Pinedo G, Liebendörfer M and Frekers D 2004 Nucl. Phys. A 777395

[38] Liebendörfer M 2005 Astrophys. J. 6331042

[39] Rampp M and Janka H-T 2000 Astrophys. J. Lett. 539 L33

[40] Shibata M and Sekiguchi Y-I 2003 Phys. Rev. D 68104020

[41] Komatsu H, Eriguchi Y and Hachisu I 1989 Mon. Not. R. Astron. Soc. 237355

[42] Woosley S E, Heger A and Weaver T A 2002 Rev. Mod. Phys. 741015

[43] Heger A, Langer N and Woosley S E 2000 Astrophys. J. 528368

[44] Heger A, Woosley S E and Spruit H C 2005 Astrophys. J. 626350

[45] Schutz B F 1985 A First Course in General Relativity (Cambridge: Cambridge University Press)

[46] Ott C D et al 2006 Astrophys. J. Suppl. 164130

[47] Woosley S E and Heger A 2006 Astrophys. J. 637914

[48] Flanagan É É and Hughes S A 1998 Phys. Rev. D 574535

[49] Miller M, Gressman P and Suen W-M 2004 Phys. Rev. D 69064026

[50] Cook G B, Shapiro S L and Teukolsky S A 1996 Phys. Rev. D 535533

[51] Tohline J E 1984 Astrophys. J. 285721

[52] Hix W R et al 2003 Phys. Rev. Lett. 91201102

[53] Dimmelmeier H et al 2007 Phys. Rev. Lett. submitted Dimmelmeier H et al 2007 Preprint astro-ph/0702305

[54] Baiotti L et al 2007 Phys. Rev. D 75044023

[55] Saijo M and Yoshida S 2006 Mon. Not. R. Astron. Soc. 3681429

[56] Ou S and Tohline J E 2006 Astrophys. J. 6511068

[57] Shibata M, Karino S and Eriguchi Y 2003 Mon. Not. R. Astron. Soc. 343619

[58] Zink B et al 2006 Phys. Rev. D submitted Zink B et al 2006 Preprint astro-ph/0611601

[59] Ott C D et al 2005 Astrophys. J. Lett. 625 L119

[60] Watts A L, Andersson N and Jones D I 2005 Astrophys. J. Lett. 618 L37

[61] Shoemaker D 2006 private communication 